1 Vorwort

Schmoll, H.-J. (Halle/Saale)

Einleitung

2 Oxaliplatin: Hohe Aktivität in allen Stadien des Kolonkarzinoms

Interview mit A. de Gramont (Paris)

11 Jetzt auch in der adjuvanten Therapie: FOLFOX4 effektiver als die Standardtherapie

\title{
ASCO-Abstracts
}

\section{Breast Cancer}

13 Cancer Prevention

14 Developmental Therapeutics - Cytotoxic Chemotherapy

14 Developmental Therapeutics - Molecular Therapeutics

15 Gastrointestinal Cancer

40 Genitourinary Cancer

41 Gynecologic Cancer

42 Head and Neck Cancer

43 Health Services Research

43 Hematologic Malignancies

44 Lung Cancer

44 Patient Care

45 Pediatric Cancer

46 Tumor Biology / Immunobiology / Human Genetics

$47 \quad$ Author Index 